\title{
Onset and recurrence of proliferative vitreoretinopathy in various vitreoretinal diseases
}

\author{
Holger Mietz, Klaus Heimann
}

Department of

Ophthalmology, University of Cologne, Germany

H Mietz

K Heimann

Correspondence to:

H Mietz, Department of

Stelzmann-Strasse 9, 50924

Köln, Germany.

Accepted for publication

17 May 1995
Ophthalmology, Joseph-

\begin{abstract}
Aims-This study aimed to evaluate both the mean time intervals between retinal disease and the development of proliferative vitreoretinopathy (PVR) and the time intervals at which recurrent PVR develops following various kinds of vitreoretinal disease and surgery.
\end{abstract}

Methods-One hundred and thirty six consecutive cases of PVR that were seen and operated on between 1991 and 1994 were evaluated retrospectively. Intervals between onset of disease and PVR or recurrent PVR were noted. Conditions leading to PVR, surgical procedures, and the final anatomical and functional results were evaluated.

Results-Before PVR was noted for the first time, an average of 1.06 vitreoretinal surgical procedures were performed (range 0 to 3). A mean of 1.99 surgeries were performed to control PVR or to reach an end stage where further surgery would have been unreasonable (range 0 to 5). The average (median) time interval between the onset of the retinal disease and PVR was 2.0 months (range 0.5 to 45

Table 1 Diagnoses of 136 consecutive cases leading to proliferative vitreoretinopathy

\begin{tabular}{lrr}
\hline Diagnosis & $n$ & $\%$ \\
\hline Aphakia & 5 & 4 \\
Pseudophakia, PC/IOL & 27 & 20 \\
Pseudophakia, AC/IOL & 1 & \\
Retinal detachment & 8 \\
Rhegmatogenous, no surgery & 39 \\
Rhegmatogenous, buckling procedure, cryotherapy & 7 \\
Rhegmatogenous, vitrectomy, gas & 3 \\
Rhegmatogenous, cryotherapy only & 1 \\
Rhegmatogenous, vitrectomy, silicone oil & 1 \\
Rhegmatogenous, vitrectomy, silicone oil, daunomycin & 1 \\
Myopia, giant tear, vitrectomy, silicone oil & 1 \\
Myopia, giant tear, vitrectomy, silicone oil, daunomycin & 1 \\
Full thickness macular hole, buckling procedure & 1 \\
After secondary PC/IOL implantation & 2 \\
Central retinal vein occlusion, traction retinal detachment & 2 \\
Branch retinal vein occlusion, traction retinal detachment & 4 \\
Uveitis & 1 \\
Uveitis, retinal necrosis, vitrectomy, silicone oil & 1 \\
Buphthalmus, vitrectomy & 1 \\
Retinopathy of prematurity, buckling procedure & 2 \\
Endogenous endophthalmitis & 1 \\
Vitreoretinal surgery for an impending macular hole & 2 \\
Trauma & 3 \\
Contusion & 2 \\
Contusion, giant retinal tear, vitrectomy, silicone oil & 4 \\
Intraocular foreign body, vitrectomy & 3 \\
Peraocular foreign body, vitrectomy, silicone oil & 7 \\
Perforating injury, no intraocular foreign body & 1 \\
Ruptured globe & 3 \\
Ruptured globe, vitrectomy, silicone oil & 1 \\
von Hippel's disease & 2
\end{tabular}

$\mathrm{AC} / \mathrm{IOL}=$ anterior chamber intraocular lens; $\mathrm{PC} / \mathrm{IOL}=$ posterior chamber intraocular lens. months). The median time intervals between surgery and recurrence for the second, third, or fourth times was also $2 \cdot 0$ months (range 0.5 to 34 months).

Conclusions-PVR starts earliest at 2 weeks after an event and subsequently quietens down within a maximum of 45 months after treatment (median 2 months). PVR may recur more than once but the time course of the disease is likely to be the same for each recurrence.

(Br f Ophthalmol 1995; 79: 874-877)

It is well established that proliferative vitreoretinopathy (PVR) is the main reason for failures of vitreoretinal surgery. ${ }^{1}$ The term PVR comprises a variety of different intraocular cellular proliferations including epiretinal membranes, subretinal strands, and retinal detachments in combination with star folds, vitreous traction, and anterior loop traction (anterior PVR)..$^{2-4}$

The clinical course of PVR has been only rarely evaluated in larger series. It is known that PVR occurs in up to $20 \%$ of cases following various vitreoretinal surgical procedures, ${ }^{5}$ and the process of PVR may also begin without any apparent cause such as idiopathic epiretinal membranes. In this study, we aimed to evaluate at what time PVR develops primarily following different kinds of vitreoretinal surgical procedures, and at what time intervals repeat PVR occurs.

\section{Materials and methods}

We retrospectively evaluated consecutive charts of all patients undergoing surgery for PVR in our department between January 1991 and January 1994. Only those charts that contained sufficient information for statistical evaluation were selected. In particular, the history of onset of retinal disease for example, retinal detachment, had to consist of sufficient exact information. Patients were followed regularly in our outpatient department, by the local ophthalmologist, or, as in most cases, by a combination of both. Intervals ranged from 1 to 4 weeks. A total of 136 cases out of 1123 fulfilled these criteria. The main reason for excluding patients from the study was that the exact time point of the onset of the retinal disease, 
Table 2 Number of vitreoretinal surgical procedures before the development of proliferative vitreoretinopathy (average, 1.06)

\begin{tabular}{llr}
\hline Number of surgical procedures & Cases $(n)$ & $\%$ \\
\hline 0 & 23 & 17 \\
1 & 87 & 64 \\
2 & 20 & 15 \\
3 & 6 & 4 \\
\hline
\end{tabular}

Table 3 Number of surgical procedures performed in each case $(n=136)$ to control proliferative vitreoretinopathy (average, 1.99)

\begin{tabular}{lcr}
\hline Number of surgical interventions & Cases $(n)$ & $\%$ \\
\hline 0 & 1 & 1 \\
1 & 55 & 40 \\
2 & 37 & 27 \\
3 & 31 & 23 \\
4 & 11 & 8 \\
5 & 1 & 1 \\
\hline
\end{tabular}

or that the exact time of onset of PVR were not clearly documented. In many cases, patients were referred from the local ophthalmologist with recurrent PVR, and the exact time point of onset was unclear.

The group consisted of $\mathbf{4 2}$ females and 94 males. All patients were white. The average age of the females at the time of the first surgery for PVR was $60 \cdot 1$ years (range 25 to 82 years); the average age of the males at the time of their first surgery for PVR was $49 \cdot 7$ years (range 7 to 81 years).

The charts were evaluated for the time course of PVR and the incidence of recurrence following surgical procedures which are routinely used today. We did not evaluate charts before 1991 when some of today's refined techniques were not available. As a consequence of this, the follow up was limited in some of the cases. The surgical techniques routinely used included scleral buckling procedures in combination with cryotherapy or laser photocoagulation, pars plana vitrectomy, the use of heavy liquids (perfluorcarbon liquid, PFCL), the use of daunomycin, endolaser photocoagulation, mobilisation of the retina, retinectomies and retinotomies, and intraocular silicone oil tamponades which were usually intended to be only temporary.

We believe that practically all patients who developed complications were referred back to our department as there are only few options in the area.

\section{Results}

The different reasons which led to PVR in the cases evaluated are listed in Table 1.

In most cases $(83 \%)$, vitreoretinal procedures had been performed before the development of PVR. In $64 \%$, one surgical procedure had been carried out before the complication

Table 4 Time intervals between trauma and occurrence of proliferative vitreoretinopathy (PVR) and between surgery for PVR and recurrent PVR

\begin{tabular}{llll}
\hline Time interval between & Median (months) & Number & Range (months) \\
\hline Retinal disease and primary PVR & $2 \cdot 0$ & 136 & $0 \cdot 5-45$ \\
First surgery for PVR and recurrent PVR & $2 \cdot 0$ & 82 & $0 \cdot 5-27$ \\
Second surgery for PVR and recurrent PVR & $2 \cdot 0$ & 33 & $0 \cdot 5-34$ \\
Third surgery for PVR and recurrent PVR & $2 \cdot 0$ & 13 & $1-5$ \\
Fourth surgery for PVR and recurrence & $2 \cdot 0$ & 1 & $2 \cdot 0$ \\
\hline
\end{tabular}

of PVR and a maximum of three procedures was noted in a total of six cases (4\%) (Table 2).

To control PVR or to reach an end stage where no further surgical intervention was considered, an average of 1.99 surgical procedures were performed (Table 3). In one case no surgery was necessary because a peripheral localised epiretinal proliferation was present with a circumscribed small retinal detachment, without a retinal hole, that did not progress over time. A single surgical procedure was sufficient to control PVR in $40 \%$ of the cases, a second major procedure had to be performed in $27 \%$; a total of three to five procedures were carried out less frequently.

The time intervals between the occurrence of the retinal disease or a surgical procedure and the development or recurrence of PVR are listed in Table 4 and shown graphically in Figures 1 to 4 . The minimum time interval was 0.5 months. The development of primary PVR occurred in a range between 0.5 and 45 months. However, within the first 3 months, $79 \%$ of PVR developed, and $90 \%$ within the first 6 months. The statistical median time intervals between onset of retinal disease and primary PVR as well as the time intervals between surgery for PVR and the recurrences was always $2 \cdot 0$ months. This may represent the time after surgery when there is the highest risk for development of PVR.

Selecting only those cases with a rhegmatogenous retinal detachment $(n=104)$, the median time intervals were similar (Table 5).

For the statistical evaluation of the average time intervals between onset of retinal disease or surgery and PVR or recurrent PVR, we calculated the median rather than the mean, because the distribution of the individual time intervals did not follow a Gaussian distribution.

The average follow up after the last surgery in each case was a median of 4 months (range 2-61 months). This time was relatively short in some cases, because we included in this study only those patients who had been recently operated on with the most advanced surgical techniques.

Both the severity of cases in this study and the difficulties of treatment of PVR may be reflected by the fact that an average of two surgical procedures were necessary either to successfully control PVR or to reach an end stage where no further surgical intervention seemed favourable (Table 2 ). In only one case was a circumscribed traction retinal detachment caused by PVR stable, and no surgery was performed. In most cases up to three major surgical procedures were necessary (23-40\%). In a few cases more surgical procedures were performed, with a maximum of five. The number of procedures was higher in those cases where the retinal situation was first stabilised with silicone oil, and a recurrent detachment followed the removal of the silicone oil after a period of time.

\section{Discussion}

Most clinical studies available state only that PVR develops following retinal detachment or 


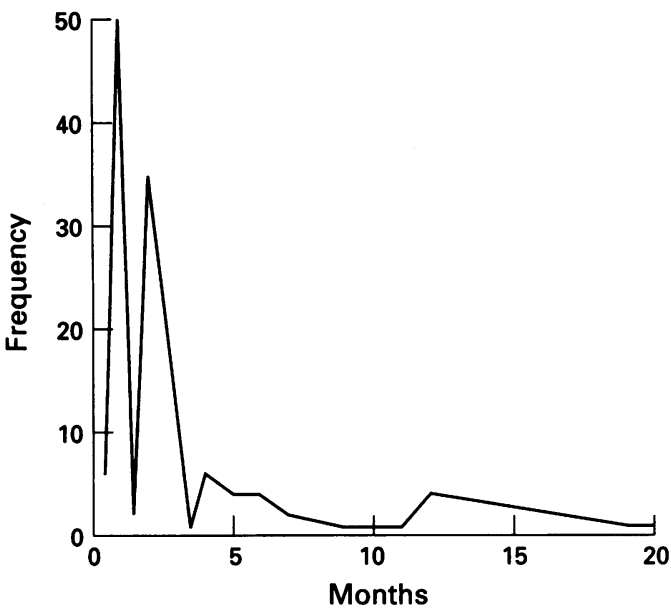

Figure 1 Frequency of primary proliferative vitreoretinopathy in relation to the time course $(n=135)$.

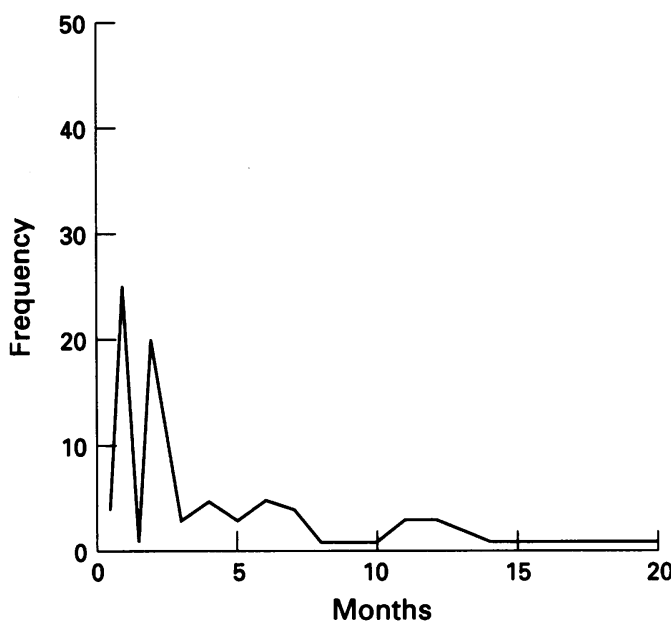

Figure 2 Frequency of recurrent proliferative vitreoretinopathy in relation to the time course $(n=82)$.

after surgery for retinal detachment, but not the exact time course of the disease. ${ }^{167} \mathrm{We}$ were interested to know whether the second or third recurrence of PVR follows a different time frame, or whether the disease process is always similar once it has started. This could be important in cases where further surgical procedures are planned - for example, secondary implantation of intraocular lenses. ${ }^{8}$

Most of the situations in which PVR developed in this study were cases after surgery for rhegmatogenous retinal detachments, performing scleral buckling procedures and cryotherapy, pseudophakic retinal detachments, and rhegmatogenous retinal detachments which had not yet been operated on. The list of diagnoses leading to PVR is long, and also rare cases like von Hippel's disease and uveitis were included (Table 1). Interestingly,

Table 5 Time intervals between trauma and occurrence of proliferative vitreoretinopathy (PVR) and between surgery for PVR and recurrent PVR for cases with rhegmatogenous retinal detachment only

\begin{tabular}{llrl}
\hline Time interval between & Median (months) & Number & Range (months) \\
\hline Onset of retinal disease and primary PVR & $2 \cdot 0$ & 104 & $0.5-45$ \\
First surgery for PVR and recurrent PVR & $2 \cdot 0$ & 63 & $0.5-22$ \\
Second surgery for PVR and recurrent PVR & $2 \cdot 0$ & 22 & $1-6$ \\
Third surgery for PVR and recurrent PVR & $2 \cdot 0$ & 9 & $1-5$ \\
Fourth surgery for PVR and recurrence & $2 \cdot 0$ & 1 & 2 \\
\hline
\end{tabular}

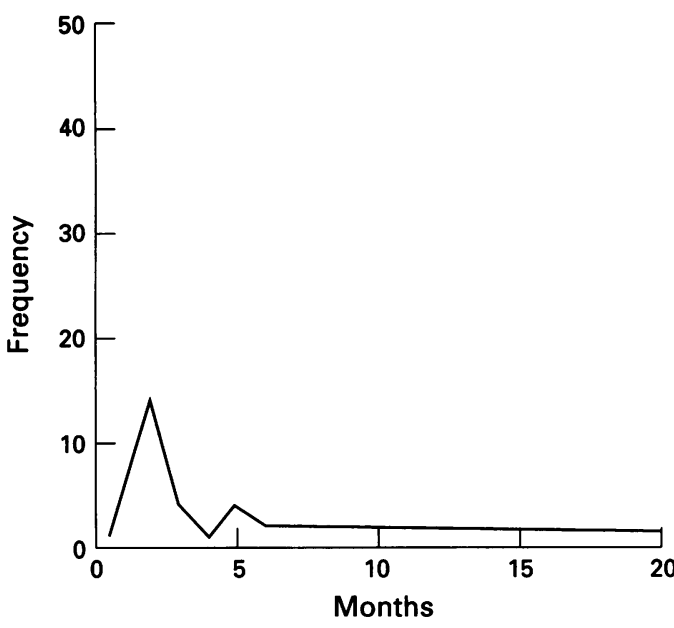

Figure 3 Frequency of proliferative vitreoretinopathy recurring for the second time in relation to the time course $(n=33)$.

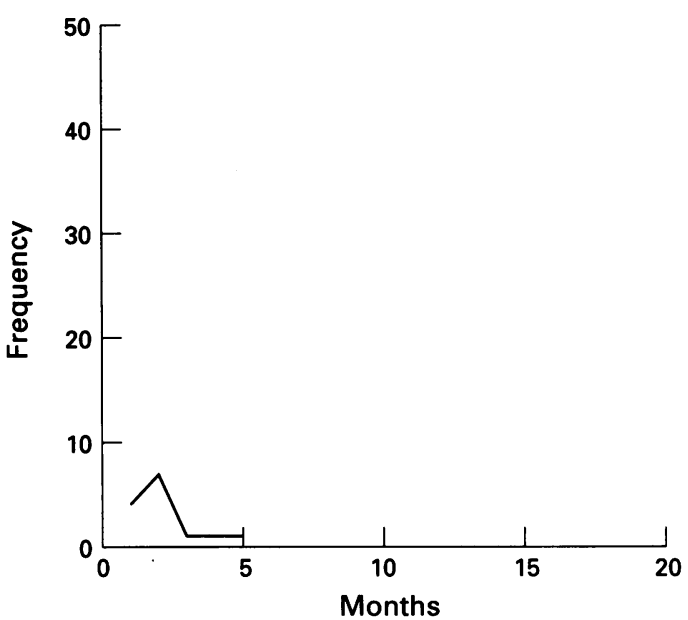

Figure 4 Frequency of proliferative vitreoretinopathy recurring for the third time in relation to the time course $(n=13)$.

the number of surgical procedures before the development of PVR was variable. While in some cases there was no preceding surgery, there were up to three surgical procedures to treat retinal detachment before the complication of PVR. In the majority of cases $(64 \%)$, PVR developed after the first vitreoretinal surgical procedure (Table 2).

The time intervals between the onset of the retinal disease and the occurrence of PVR were similar in all cases. The concordance of these results is surprising given the large number of cases and heterogeneity of underlying diseases. In a few of the cases where PVR developed for the first or second time, the time intervals were long with a time lapse of up to 45 and 27 months, respectively, It may be speculated that in these instances where PVR developed after more than 12 months, a new stimulus caused the recurrence rather than a recurrence related to the original disease after that time. However, we do not have enough data to prove this assumption, although it seems very likely.

In summary, judging from these results, PVR develops in the vast majority within the first 2 months both after onset of retinal disease and for recurrences. It seems that these recurrences follow a similar time frame as the 
first episode. Factors predisposing to recurrences may therefore be the same as for the primary occurrence - for example, retinal tears, extensive cryotherapy or laser therapy, retinal ischaemia, and others. ${ }^{9} 10$

From the current models of PVR which explain this process by cellular proliferations along one or both surfaces of the retina or along the anterior vitreous base (anterior PVR), it can be assumed that PVR can not develop directly following a stimulus, because the cells involved need time for migration, transformation, proliferation, and subsequent contraction. ${ }^{11-17}$ The clinical data of this study therefore support these models. A development of PVR many years after onset of a specific retinal disease also seems unlikely, because the cellular proliferations have to be related to a stimulus, and is unlikely to start without a reason. The time course of recurrent PVR was similar to that of primary PVR in our study and again supports the theoretical assumption that the underlying process of primary and recurrent PVR is similar.

Since our study was performed retrospectively, conclusions from the data have to be drawn with much care. However, the information from the charts in the cases that were entered into this investigation was well documented, so reliability of the data discussed can be assumed.

1 Machemer R. Massive periretinal proliferation: a logical approach to therapy. Trans Am Ophthalmol Soc 1977; 75: approach

2 Lopez PF, Grossniklaus HE, Aaberg TM, Sternberg PJ, Capone AJ, Lambert HM. Pathogenetic mechanisms in anterior proliferative vitreoretinopathy. Am $\mathcal{f}$ Ophthalmol 1992; 114: 257-79.

3 Aaberg TM. Management of anterior and posterior proliferative vitreoretinopathy. XIV. Edward Jackson memorial lecture. Am ₹ Ophthalmol 1988; 106: 519-32.

4 Lewis H, Aaberg TM. Anterior proliferative vitreoretinopathy. Am f Ophthalmol 1988; 105: 277-84.

5 Ryan SJ. Traction retinal detachment. XIIX Edward Jackson memorial lecture. Am $\mathcal{F}$ Ophthalmol 1993; 115: 1-20.

6 Smiddy WE, Maguire AM, Green WR, Michels RG, Cruz $\mathrm{Z}$ de la, Enger $\mathrm{C}$, et al. Idiopathic epiretinal membranes. Ultrastructural characteristics and clinicopathologic correlation. Ophthalmology 1989; 96: 811-21.

7 Wiedemann P, Leinung C, Hilgers RD, Heimann $\mathrm{K}$ Daunomycin and silicone oil for the treatment of proliferative vitreoretinopathy. Graefes Arch Clin Exp liferative vitreoretinopathy.

8 Mietz H, Konen W, Heimann K. Visual outcome of secondary lens implantation after trauma or complicated retinal detachment surgery. Regina 1994; 14: 212-8.

9 Bonnet M. The development of severe proliferative vitreoretinopathy after retinal detachment surgery. Grade B: a determining risk factor. Graefes Arch Clin Exp Ophthalmol 1988; 226: 201-5.

10 Bonnet $\mathrm{M}$, Hajjar $\mathrm{Ch}$. Clinical risk factors for proliferative vitreoretinopathy. In: Strippe A, ed. Advances in vitreoretinal surgery. Brucker A. Ophthalmic communications retinal surgery. Brucker A. Op
Soc: New York, 1992: 229-36.

11 Glaser BM, Vidaurri-Leal J, Michels RG, Campochiaro PA. Cryotherapy during surgery for giant retinal tears and intravitreal dispersion of viable retinal pigment epithelial cells. Ophthalmology 1993; 100: 466-70

12 Weller M, Heimann $\mathrm{K}$, Wiedemann P. Immunochemical studies of epiretinal membranes using APAAP complexes: evidence for macrophage involvement in traumatic proliferative vitreoretinopathy. Int Ophthalmol 1988;11: 181-6.

13 Hiscott PS, Grerson I, McLeod D. Retinal pigment epithelial cells in epiretinal membranes: an immunohistochemical study. Br $f$ Ophthalmol 1984; 68: 708-15.

14 Yeo JH, Sadeghi J, Campochiaro PA, Green WR, Glaser YM. Intravitreous fibronectin and platelet-derived growth
BM, Sader factor. New model for traction retinal detachment. Arch Ophthalmol 1986; 104: 417-21.

15 Heidenkummer HP, Kampik A. Vergleichende immunhistochemische Untersuchungen epiretinaler Membranen bei proliferativen vitreoretinalen Erkrankungen. Fortschr Ophthalmol 1991; 88: 219-24.

16 Wiedemann P. Growth factors in retinal diseases: proliferative vitreoretinopathy, proliferative diabetic retinopathy, tive vitreoretinopathy, proliferative diabetic retinopathy, and retina

17 Green WR. Vitreomacular juncture. In: Ryan SJ, ed. Retina. Vol 3, Ch 117. St Louis: Mosby, 1989: 13-69. 